\title{
Pétitions de femmes en faveur d'une réforme révolutionnaire de la famille
}

\section{Suzanne Desan}

\section{(2) OpenEdition \\ 1 Journals}

\section{Édition électronique}

URL : https://journals.openedition.org/ahrf/5883

DOI : 10.4000/ahrf.5883

ISSN : 1952-403X

Éditeur :

Armand Colin, Société des études robespierristes

\section{Édition imprimée}

Date de publication : 1 juin 2006

Pagination : 27-46

ISSN : 0003-4436

\section{Référence électronique}

Suzanne Desan, « Pétitions de femmes en faveur d'une réforme révolutionnaire de la famille », Annales historiques de la Révolution française [En ligne], 344 | avril-juin 2006, mis en ligne le 01 juin 2009, consulté le 23 avril 2022. URL : http://journals.openedition.org/ahrf/5883 ; DOI : https://doi.org/ 10.4000/ahrf.5883

Ce document a été généré automatiquement le 23 avril 2022.

Tous droits réservés 


\title{
Pétitions de femmes en faveur d'une réforme révolutionnaire de la famille
}

\author{
Suzanne Desan
}

\section{NOTE DE L'ÉDITEUR}

Traduit par Françoise Basch, professeur émérite de civilisation anglo-américaine, université de Paris-VII Denis Diderot.

1 Au printemps 1795, la citoyenne Heringue adressait un appel angoissé à la Convention nationale lui demandant instamment de soutenir la loi du 17 nivôse an II, qui garantissait le partage égal de l'héritage, "préservant ainsi la paix entre frères et sœurs ». L'abrogation de cette loi, prévient-elle, priverait des milliers d'individus des droits naturels, imprescriptibles et inaliénables, reconquis le 14 juillet 1789. Et qu'arriverait-il, demande-t-elle, à celles de nos sœurs qui viennent de recevoir la part qui leur revient? La citoyenne Lefranc appuya énergiquement cet appel en insistant sur la réaction de colère de celles qui se verraient spoliées de leurs nouveaux droits sur l'héritage. «Elles vous diraient avec raison que vous êtes injustes et profitez de ce que nous ne sommes pas représentées à la Convention [...] elles vous diraient enfin que ce sont les hommes qui ont fait les lois, ils les ont faites pour eux $»^{1}$. Heringue et Lefranc n'étaient pas les seules Françaises à placer les questions d'égalité de genre et de réforme de la famille au centre de l'expérience vécue de la Révolution par maintes d'entre elles. Elles n'étaient pas non plus les seules à relier leurs observations sur la famille à la question de la participation politique et des droits des femmes au sein de la république et à faire part de leurs opinions aux députés en toute candeur et simplicité.

2 Tout au long de la Révolution, des femmes et aussi des hommes venant de tout le pays, assaillent les assemblées nationales successives de pétitions exigeant des modifications $\mathrm{du}$ droit de la famille, droit qui s'appliquait à des domaines aussi divers que 
l'illégitimité, le statut des enfants naturels, l'adoption, l'autorité parentale et les mariages arrangés. Le plus souvent, les pétitionnaires s'intéressaient à des sujets controversés: l'égalité de l'héritage et le divorce ${ }^{2}$. La loi du 20 septembre 1792 autorisait les époux à demander conjointement le divorce, soit par consentement mutuel, soit par l'une des parties pour cause d'incompatibilité, de mauvais traitements et de désertion du domicile conjugal. Dans le domaine de l'héritage, on constate que les législateurs promulguent des lois de plus en plus égalitaires. Après l'abrogation de la primogéniture en 1790, l'Assemblée nationale décrète en avril 1791, que les successions sans testament sont à répartir également entre tous les enfants sans considération de sexe ni d'âge. En 1793 et 1794, la Convention étend ce principe égalitaire à toutes les formes d'héritage en ligne directe et collatérale: les députés votent des décrets ${ }^{3}$ qui stipulent que l'ensemble des descendants, y compris les enfants naturels reconnus par leurs parents, doivent se répartir également les biens de la famille. Cette division égalitaire devait s'appliquer rétroactivement - ce qui n'alla pas sans discussion - à partir de juillet 1789, de par la loi du 17 nivôse an II (6 janvier 1794). Ces nouvelles pratiques relatives au divorce et à l'égalité de l'héritage, qui frappaient au cœur de la famille, des attentes des individus, de leurs dispositions sur leurs biens et des coutumes agraires, donnèrent lieu à d'âpres et intenses controverses.

Cet article examine les pétitions féminines concernant la famille, notamment l'héritage et le divorce, afin d'analyser l'activisme politique des femmes et leur rapport à l'État, afin aussi de participer au débat historiographique quant aux effets de la Révolution sur le genre et les femmes. Mon propos est enfin de mieux comprendre comment la Révolution remodela la famille. Selon un important courant historiographique, la Révolution en excluant les femmes de la politique et en les assignant à la sphère privée, aurait jeté les bases du concept de domesticité. On a également dit que la politique libérale ou républicaine reposait sur une logique d'exclusion des femmes et de leur subordination au moyen d'un "contrat sexuel $»^{4}$. Mais celles qui adressaient leurs pétitions aux législateurs en vue de réformer la famille démentent cette interprétation. Notre article démontre en effet que la Révolution, loin d'assigner à la famille une place à part comme « sphère privée ", n'encourageait pas uniquement le rôle domestique des femmes. Je dirais plutôt que la Révolution érigeait le statut de la famille en terrain de contestation politique : les individus voyaient les principes audacieux de la politique révolutionnaire s'appliquer au domaine personnel et ils n'hésitaient pas à s'engager dans des activités politiques: envoyer par exemple des pétitions à leurs députés ou porter leurs litiges devant les tribunaux, toutes activités propres à relier le privé au public et à l'État.

4 En outre, la Révolution produisit des effets multiples et parfois contradictoires sur la situation des femmes au sein de la famille et de l'État, soumettant ainsi à la controverse la question des relations de genre. D'autre part, il ne fait pas de doute que les chefs révolutionnaires incitaient les femmes - en tant qu'épouses et mères républicaines - à jouer un rôle patriotique dans leur foyer, mais que d'autre part, elles se voyaient accorder des droits civiques accrus à l'intérieur de la famille. La loi qui légalisait le divorce et l'égalité dans la succession ainsi que l'affaiblissement de l'autorité parentale, perturbait la dynamique de la famille et défiait souvent l'autorité traditionnelle des maris et des pères. Les nouvelles pratiques révolutionnaires encourageaient sœurs, mères, épouses et filles à porter leurs revendications personnelles dans l'arène plus 
vaste de l'État. L'idéologie des droits et de l'égalité leur fournit de nouveaux outils aptes à critiquer l'inégalité des sexes au foyer.

Femmes et pétitions

5 Les pétitions adressées aux diverses assemblées révolutionnaires, ou individuellement aux députés et aux comités, nous donnent un précieux aperçu de la façon dont l'individu ordinaire percevait et accueillait les réformes de la famille et de ses rapports à la nouvelle politique et au nouvel État. La majorité des pétitionnaires, particulièrement à partir de 1792, étaient impliqués dans des contentieux. Plusieurs de ceux et celles qui figurent dans cet article étaient simultanément en procès de divorce ou d'héritage. Leurs propos reflètent bien les préoccupations, les impressions, et les opinions politiques diverses de ceux qui tentaient de réformer la famille. Conçues comme moyen de persuasion, les pétitions demandent à être lues attentivement, les auteurs s'adonnant parfois à une rhétorique outrancière et enflammée. Engagés dans litiges et procès, il leur arrivait d'exagérer ou de déformer les effets des lois révolutionnaires sur la dynamique familiale. Pourtant même quand, dans leurs écrits, ils posent la nécessité d'une action réciproque entre la famille, la Révolution et la loi, la vision utopique qui est la leur nous permet de comprendre les préoccupations et programmes politiques populaires.

6 Les pétitions des femmes portant sur le droit de la famille variaient largement quant à la forme et au style d'argumentation. Établir la paternité du texte est essentiel à l'analyse des pétitions. Les plus répandues sont des textes manuscrits émanant d'individus. Parfois on constate, dans une dizaine de cas, que les femmes signaient des pétitions rédigées par des hommes de loi, un mari ou tout autre mandataire. Je n'ai pas trouvé d'exemple de pétitionnaire recopiant un modèle de pétition en faveur du divorce ou du droit des femmes à hériter ${ }^{5}$. Tout semble indiquer que la grande majorité d'entre elles rédigeaient leurs propres requêtes. La plupart de ces pétitions féminines étaient mal écrites, gribouillées, à peine lisibles et fourmillaient de fautes de grammaire et d'orthographe. Certaines de ces correspondances étaient remarquablement mal informées et réagissaient à des rumeurs ou des interprétations erronées de lois nouvelles et complexes. D'autres pétitionnaires, bien que presque illettrées, manifestaient une étonnante compréhension des lois anciennes et nouvelles, car la presse révolutionnaire les tenait informées des débats législatifs et de l'opinion dans de lointaines régions. Par exemple, explique la citoyenne Leblanc: "J'ai vu dans un journal que toute femme divorcée était libre de se remarier au bout de dix mois». Angélique Fouque rapporte qu'apprenant dans la presse que, selon la loi du 4 floréal an II, le divorce pouvait être prononcé après une séparation de six mois, elle décida de recourir à cette procédure plus simple et plus expéditive et de renoncer à celle qui procédait à la recherche des causes ${ }^{6}$.

7 Il arrivait que des groupes de femmes envoient des pétitions collectives concernant des questions d'héritage. Parfois imprimées, leur origine ne se laisse pas facilement repérer. Ainsi de nombreuses communes, notamment dans le Midi, envoyaient des pétitions signées par des hommes et des femmes, qui revendiquaient plus généralement un partage égal. Les frères faisaient parfois cause commune avec leurs sœurs. Ces campagnes d'origine méridionale qui revendiquent le partage égal de l'héritage pour les fils cadets et dans une moindre mesure pour les filles, générèrent davantage de pétitions collectives et d'appels sous la forme de brochures que le mouvement en faveur des droits des femmes à l'héritage ${ }^{7}$. 
8 Les pétitionnaires n'épousaient pas toutes le même point de vue sur ces questions. Certaines s'opposaient vigoureusement à la réforme de la famille. Les épouses divorcées depuis peu, par exemple, se plaignaient amèrement de la perte soudaine et inattendue de leurs biens, de leur situation et de leur statut. De même, sur la question de l'héritage, il arrivait que des mères et des épouses s'en prennent à la loi au nom de leur mari ou de leur fils récemment spolié, $\mathrm{du}$ fait $\mathrm{du}$ changement de règles régissant l'attribution de legs à des individus étrangers à la famille, l'ordre des successions collatérales, les donations entre vifs, etc. ${ }^{8}$. Puisque ma recherche porte essentiellement sur la politisation des femmes qui soutiennent la Révolution, je vais d'abord puiser dans les pétitions de femmes qui demandent des changements dans le droit privé. J'inclurai parfois des comparaisons avec des pétitions masculines sur les mêmes questions, pour situer les revendications féminines dans un contexte de litige. Je me propose d'examiner la posture morale des femmes, leur mode d'utilisation de l'idéologie révolutionnaire, leurs attentes vis-à-vis de l'état et leurs perspectives sur la réforme de la famille.

Critique du despotisme familial

9 Les femmes qui revendiquaient l'accès au divorce, le partage égal de la succession et le maintien de la loi du 17 nivôse, adoptaient la logique révolutionnaire du droit naturel à l'égalité et à la liberté. Quand, en 1795, une femme originaire du Calvados défend la rétroactivité de la loi du 17 nivôse, c'est parce que, selon elle, cette loi rétablit les femmes « dans [leurs] droits naturels et imprescriptibles [...]. L'Assemblée législative a décrété en 1789 tous les hommes égaux en droits, et que sous cette expression générique les femmes et les filles étaient comprises ». Au nom de la même cause, Jeanne Barinçon, originaire de l'Aude, regrettait qu'il eût fallu " trois ans, six mois et 22 jours " aux députés pour rétablir l'égalité des droits en instituant le partage égal de l'héritage. Il allait sans dire que la rétroactivité de ces droits devait prendre effet dès 1789 car « un grand peuple qui a tout fait pour briser ses chaînes et rétablir ses droits, doit en jouir dès l'instant qu'il s'est élevé contre les usurpations »".

10 Les pétitionnaires ne cessaient de répéter que la nature exigeait l'égalité à l'intérieur de la famille; il incombait aux lois et aux institutions politiques de rétablir ce droit naturel. Cette rhétorique était commune aux sœurs et aux frères cadets, dont les pétitions sur l'héritage étaient bien plus nombreuses. Les individus et les groupes exposaient leur situation juridique particulière comme autant d'atteintes à l'égalité que les députés se devaient de réparer. En Normandie, par exemple, au début des années 1790, une série de pétitions collectives, n'émanant que de femmes, adoptèrent cette stratégie lorsqu'elles demandaient des lois spécifiques propres à protéger ou à augmenter leur part légitime. Les "citoyennes du département du Calvados" affirmaient que leurs revendications étaient « de nature à ne pouvoir être rejetées par les défenseurs de l'Égalité", ce qui faisait écho à une déclaration similaire des « citoyennes » de Coutances en 1792. «Plusieurs filles de Saint-Lô » mettaient en garde l'Assemblée législative, lui signifiant que « l'immortelle constitution [...] fera le bonheur de tout le peuple français lorsque l'Assemblée aura seulement songé que les filles légitimaires de la ci-devant province de Normandie n'ont point encore été l'objet de votre sollicitude pour la Nation ». "La nature en naissant nous a donné des droits égaux à la succession de nos pères " affirmaient une quarantaine de femmes de la région de Falaise ${ }^{10}$. 
11 De même que la revendication du partage égal de l'héritage insistait sur le droit naturel à l'égalité, les défenseurs du divorce clamaient le droit à la liberté. En octobre 1793, la citoyenne Gavot fit cadeau de son alliance, "symbole de mon esclavage ", à la Convention nationale en témoignage de gratitude pour « la loi sainte du divorce ». Elle déclara avoir été « gémissante sous l'empire d'un mari despote [...]. Aujourd'hui rendue à la dignité de femme indépendante, elle adore cette loi bienfaisante qui rompt les nœuds mal assortis; qui rend les cœurs à eux-mêmes, à la nature, enfin à la divine liberté ». De même lorsque Geneviève Lebourdais demandait la modification des règles de résidence de la loi sur le divorce, elle faisait le commentaire suivant : " Il ne faut pas chez un Peuple Libre et Républicain qu'il existe un seul individu pour lequel le mariage soit un lien indissoluble ${ }^{11}$.

12 Même si les partisans de la réforme de la famille, hommes et femmes, adoptent un langage d'égalité, de droit, de liberté, ils utilisent une rhétorique différente dans leurs pétitions sur le divorce et la succession. Il n'est pas sans intérêt d'explorer ces différences pour mesurer à quel point la Révolution encourageait les femmes à mettre en question la "dynamique du genre» dans la famille et, dans un certain sens, stimulait leur conscience politique.

13 Premier exemple: le divorce. Dans leurs pétitions, les hommes comme les femmes imaginaient le divorce comme une façon de se libérer de mariages à problèmes et sans amour ; mais dans les pétitions masculines, la rhétorique de l'émancipation s'exprime d'une manière plus abstraite. « Une journée d'esclavage est une journée de deuil pour la société et une journée de mort pour un patriote », proclamait Gauthier en demandant qu'on abrège le laps de temps requis avant le remariage. Les hommes avaient recours à des images de " chaînes » ou de "fers " qui les rendaient « esclaves de l'Hymen ", les contraignaient à des mariages de convenance ou leur interdisaient de se remarier. Furieux contre une épouse rebelle, le citoyen Robin se plaignait de vivre «dans une sorte d'esclavage ", ajoutant que, si le divorce n'existait pas, "nous serions deux êtres antisociaux infiniment pour ne pas dire éternellement malheureux ». Les hommes ont davantage tendance à considérer le mariage comme un «contrat qui peut être dissous par la volonté des deux parties » et à insister sur la nécessité de pouvoir se libérer du mariage plutôt que d'en réformer la dynamique interne ${ }^{12}$.

En revanche, les femmes pétitionnaires étaient plus explicites sur la tyrannie exercée à l'intérieur $\mathrm{du}$ mariage, montrant $\mathrm{du}$ doigt la domination masculine ou tel abus spécifique pour justifier leur désir de divorce ou de réforme du divorce. Leurs pétitions se faisaient l'écho des franches critiques du « despotisme marital » exprimées au début de la Révolution par les auteurs féministes de pamphlets, de cahiers et de traités de morale ${ }^{13}$. Engagée dans une pénible procédure de divorce, la citoyenne Le Machais comparait sa cause à «celles de toutes les victimes que vous avez voulu soustraire à l'oppression [...]. Vous nous avez promis la liberté, vous n'avez pas prévu sans doute tous les moyen que nos ci-devant maîtres emploieraient pour conserver leur empire despotique ». Tandis que la citoyenne Andaye contait comment son mari avait dilapidé sa fortune, Félicité Desrouges se répandait amèrement sur les trois années de séquestration dans un couvent, acte perpétré par son mari. Plus optimiste, une autre pétitionnaire soutenait que la possibilité de divorcer pourrait bien « rappeler à certains hommes (ce qu'ils avaient oublié) à savoir qu'une femme n'était pas leur esclave, mais bien leur compagne et qu'elle ne devait pas être la victime de leurs humeurs et mauvais traitements $»^{14}$. Pour résumer, bien des femmes partageaient avec les hommes l'espoir 
d'un mariage basé sur la bonne entente, mais accusaient plus directement la hiérarchie conjugale qui mettait en péril cette harmonie.

Même si les hommes comme les femmes cherchaient un remède pour échapper aux malheurs conjugaux, ce sont les femmes qui souffraient le plus directement du déséquilibre des rapports de pouvoir au sein du mariage. Il ne fait pas de doute que, dans la vie quotidienne, la relation entre l'autorité du mari et la soumission de la femme faisait l'objet de négociations constantes. Il arrivait que les femmes exercent un pouvoir inhabituel et que certains époux acceptent de transiger sur leur autorité, au fur et à mesure que se créaient entre eux des rapports de dépendance et d'estime mutuelle. Pourtant, l'ensemble des lois, de la culture populaire, des structures économiques et des stratégies familiales, renforçait la domination masculine dans la famille. Repliée à l'intérieur d'un mariage difficile, une femme avait moins de choix que son mari. Des époux divorcés des deux sexes exprimaient leur aspiration au bonheur conjugal et leur frustration de par le manque de communication intime et affective; mais les pétitions des épouses à la Convention et à la justice invoquaient pareillement le manque d'affection et d'autres problèmes comme l'abandon du domicile conjugal, l'appauvrissement et les mauvais traitements. On remarque que, dans leurs récits des défauts des époux, les femmes mettent en œuvre une approche pragmatique plutôt que sentimentale. Impatiente d'échapper au "joug de l'esclavage», la citoyenne Van Houten déplorait un mariage arrangé avec « un homme emporté, tracassier, stupide, malpropre et paresseux [...] et de l'incapacité la plus absolue en affaires $»^{15}$.

De même que les femmes font état d'une gamme plus étendue et plus spécifique de problèmes conjugaux que les hommes, elles prennent plus souvent l'initiative du divorce devant les tribunaux et leur décision intervient à partir d'un ensemble plus vaste de difficultés maritales. En France, environ deux tiers des procédures de divorce initiées par l'une des parties provenaient des femmes, $72 \%$ dans le Calvados par exemple. Je constate dans mon analyse statistique des divers motifs de divorce dans cette région, que lorsque le mari prend l'initiative, il invoque généralement l'une des deux causes suivantes : le plus souvent, l'incompatibilité d'humeur ou diverses formes d'absence et de désertion du domicile conjugal. Telles étaient aussi les catégories qui revenaient le plus souvent pour les femmes : en Basse-Normandie, $73 \%$ des demandes de divorce pour incompatibilité sont le fait des femmes contre $71 \%$ pour cause d'abandon ou d'absence. Mais, en dehors de ces motifs et de quelques accusations d'immoralité, les hommes invoquent rarement des causes spécifiques de divorce. En revanche, dans l'ensemble des demandes de divorce pour raisons diverses, à la requête d'une des parties, ce sont les femmes qui dominent, sauf pour l'accusation d'immoralité. On constate le même comportement à Lyon, Paris, Rouen et Metz ${ }^{16}$. En 1795, la citoyenne Jacquette dresse un inventaire des problèmes susceptibles de pousser une femme au divorce: "Pour sauver ma vie de la fureur de mon époux; pour ne pas partager l'infamie dont l'aura couvert un crime pour lequel il aura été condamné ; pour me soustraire à ses crimes, sévices ou injures graves; pour ne pas être témoin de son dérèglement; pour le punir de son ingratitude lorsqu'il aura été assez cruel de m'abandonner ; enfin pour ne pas être complice de son émigration, ou pour ne pas être tyrannisée parce que l'on prétendait tourner contre moi ce crime de mon époux; je ne trouve de salut que dans la loi du divorce $»^{17}$.

17 En somme même si les hommes comme les femmes revendiquent le divorce comme une forme de liberté, ce sont plutôt les femmes qui associent leurs demandes, et devant les 
tribunaux et dans les pétitions à la Convention, à la « critique du despotisme conjugal ». Comme le dit Catherine Bagot, «c'est surtout pour le sexe qu'on est convenu d'appeler le plus faible et qui cependant s'est montré le plus ardent pour la Révolution, c'est pour ce sexe jusqu'ici soumis aux volontés arbitraires de l'autre que le divorce est le plus nécessaire $»^{18}$.

Les pétitions en faveur de l'égalité de l'héritage déploraient aussi l'abus de pouvoir au sein de la famille. De même que maris et femmes imaginaient des modes différents de liberté conjugale, les fils et filles cadettes n'avaient pas la même conception du "despotisme» familial. Les fils soulignaient avant tout la tyrannie paternelle et prenaient rarement garde au facteur de genre qui, dans la dynamique des concepts, tels que "paternels", "fraternels " ou "despotisme conjugal », jouaient contre les sœurs et les épouses. Les rapports familiaux plus affectueux et plus justes qu'imaginaient les frères étaient tout aussi dénués de respect entre les sexes qu'auparavant. Ils se plaignent au contraire de ce que leur père castre les cadets en s'opposant à leur mariage et en ne les laissant pas disposer librement de leurs biens. Condamnés au célibat, les frères cadets se retrouvent "cruellement " chassés de la maison familiale, parfois poussés à entrer dans les ordres ou bien "contraints » de déserter "la campagne et souvent le royaume", selon les termes d'un citoyen courroucé. Quant aux fils enrôlés dans l'armée, privés de biens et d'une indépendance chèrement acquise, leur colère était intense. Conscients des nouveaux droits politiques, acquis en tant que citoyens, certains d'entre eux voyaient ce processus d'infantilisation par le père comme lourd de conséquences économiques et politiques. En l'absence de réforme de l'héritage, il était facile pour un père vindicatif et opposé à la Révolution de pénaliser dans son testament, un fils qui en était partisan. Se comparant à un serf de la région du Jura, un fils se plaignait de ce qu'il lui était impossible d'acheter ou de vendre. Il ne pouvait pas non plus profiter du fruit de son travail ; pire encore, sans le statut de propriétaire, il n'avait pas la possibilité d'être un " citoyen actif » français ${ }^{19}$. Il importait donc que les fils aussi jouissent de la qualité pleine et entière d'adulte de sexe masculin et de citoyen politique, droit qui devait faire partie intégrante du nouveau statut d'égalité dans la famille.

19 Quant aux filles, même sans imaginer que leurs revendications d'héritage leur vaudraient la pleine citoyenneté politique, elles appliquaient directement les principes révolutionnaires à leur propre situation familiale. Elles ne cessaient de dénoncer le "despotisme des pères et des frères » et de démasquer la capitulation quotidienne devant les intérêts de la famille. Deux citoyennes anonymes, originaires de Rouen ouvrirent le feu sur ce thème en 1789 avec leur « Remonstrances des mères et des filles normandes de l'ordre du tiers »: «Si l'on admet enfin, comme principe substitutif, que le plus fort n'imposera plus la loi au plus faible dans la grande famille de l'État, pourquoi la subirons-nous dans nos familles particulières ? Pourquoi resterions-nous asservies à cette coutume barbare qui permet au père de sacrifier ses filles par des mariages mal assortis, à l'ambition puérile d'enrichir celui qui portera son nom ?[...]. Sur quel motif voudrait-on maintenir ces odieuses distinctions entre des enfants qui ont un droit égal à la tendresse paternelle? $»^{20}$.

20 En Normandie et dans le Midi, les femmes développaient ces thèmes dans leurs pétitions, déclarant que la Loi elle-même, les «lois barbares » et les « coutumes tyranniques » de l'Ancien Régime «féodal» avaient détruit les rapports naturels d'affection et d'égalité dans la famille et avaient en particulier durci les cœurs 
paternels. «Les pères de Normandie [...] regardaient leurs filles comme si elles n'eussent pas été de leurs enfants »; ils les dotaient donc "suivant leur caprice ». De même, l'ancienne loi avait encouragé les frères à « rester sourds aux cris de la Nature et à la voix de la justice ». D'une pétition l'autre, les femmes qui revendiquaient l'égalité dans l'héritage se servaient de la terminologie révolutionnaire pour dénoncer les " frères dénaturés ", coupables de « long et accablant despotisme », et qui bénéficiaient $\mathrm{du}$ " privilège [...] qui blessait le droit commun de l'égalité $»^{21}$. Elles maîtrisaient aussi l'antique vocabulaire de la faim pour condamner la pauvreté des femmes. Dans un langage prosaïque, Marguerite Briançon prévenait la Convention qu'en cas d'abrogation de la loi du 17 nivôse, «mon frère sera un gros riche et moi je n'aurai pas du pain [...]. Rendez, représentants, bonne justice et faites que l'égalité entre frère et sœur soit maintenue ». D'innombrables écrits soulignaient le contraste entre la dure condition des sœurs et «l'opulence », « l'oisiveté » et « la cupidité » des frères aînés et demandaient «au nom de l'humanité » une « loi juste qui veut que tout citoyen puisse vivre $»^{22}$.

Certaines contaient leur malheur sur le ton pathétique du roman et des mémoires judiciaires pour insuffler plus de vigueur à leurs pétitions, pour mettre en lumière le caractère immoral des us et coutumes prérévolutionnaires et pour émouvoir le cœur sensible des députés ${ }^{23}$. La fille Anquetil décrivait comment son frère l'avait séquestrée, accusée de vol, comment il s'était emparé de ses biens, l'avait expulsée de la maison à minuit, puis avait fait traîner interminablement la procédure. D'autres, au moyen de descriptions plus générales, soulignaient le caractère universel des torts faits aux femmes. Les « citoyennes du Calvados » se plaignaient du despotisme qui avait régné en Normandie. À présent, «le stoïcisme de quelques amis de la liberté et de l'égalité a terrassé ce monstre [...] mais on dirait qu'il s'est réfugié dans notre département [...] il savait bien qu'il trouverait dans ce pays presque autant de protecteurs qu'il y a d'habitants mâles, puisque tous sont intéressés à conserver les prérogatives dont ils jouissaient au préjudice des filles $»^{24}$.

22 En mettant à nu la pratique courante par laquelle les filles se sacrifiaient à la lignée familiale, les pétitionnaires transposaient dans le discours, signifiaient dans la pratique, leurs litiges auprès des tribunaux de famille concernant l'héritage. Dans le Calvados par exemple, dans les années 1790, les sœurs invoquaient sans cesse la nouvelle législation sur l'héritage aux fins de gagner contre leurs frères au tribunal ${ }^{25}$. Comme cette procédure, les pétitions des femmes mettaient en question toute une structure destinée à conserver intacts les biens familiaux, à confier les décisions aux seuls pères et à faciliter l'établissement d'un ou de plusieurs fils. Selon les termes de la citoyenne Beaufils veuve Lavallée: "C'était une manie ou un préjugé de nos pères, poussé à l'excès, de favoriser la conservation des biens propres ou de familles ». Elle saluait les lois révolutionnaires qui avaient aboli les distinctions entre les biens d'origine différente et dressé des obstacles au pouvoir des familles qui entendaient conserver leurs biens au sein de la lignée paternelle ou maternelle. La citoyenne Monfreulle décrivait prosaïquement la situation : «J'ai été mariée en 1773, en terre de Normandie pour un bouquet de roses. C'est ainsi qu'on mariait les filles. La cupidité a été dans le temps et on sacrifiait souvent les filles pour le bonheur d'un seul garçon $»^{26}$. Une longue pétition imprimée s'expliquait là-dessus : "Chaque famille et surtout dans la classe des cultivateurs, désirait voir briller plusieurs de leurs enfants dans l'Église, la robe, le commerce, l'armée, ou les autres arts, et c'étaient les filles qui, par respect et par obéissance pour leur père et mère, par amitié pour leurs frères, par leur 
permanence dans la maison, par leurs soins et par leur industrie, soutenaient les travaux du père de famille, et leur économie enrichissait leurs frères ${ }^{27}$.

En associant des coutumes familiales courantes à la tyrannie et à l'injustice, les femmes insistaient sur deux thèmes chargés de signification politique et morale: le besoin d'attaquer la richesse égoïste et de retrouver des sentiments humains et naturels. Leur protestation revêtait une autorité particulière car elles alliaient jugement moral traditionnel et nouvelle éthique révolutionnaire. Leurs récits joignaient des descriptions de cupidité extrême empruntées à la culture populaire, à une imagerie jacobine qui représentait la richesse comme un crime d'un point de vue politique et éthique. Elles adoptaient en outre l'idéal républicain de fraternité et de sensibilité. Jeanne Côme, originaire de Gironde, tenait à ce sujet un discours plein de couleur : "Notre frère roule dans l'aisance, feu notre père le fit un gros procureur en la cidevant justice royale [...] un notaire tout rond et bouffi d'orgueil ». Mais auprès du lit de mort de son père, à peine lui avait-elle "fermé les paupières » et donné « cours à la Nature, les cris et les pleurs » que son frère aîné réunissait toute la famille dans une pièce attenante pour annoncer qu'il avait obtenu par la ruse que son père lui lègue tous ses biens à lui et à lui seul. C'est bien la puissance excessive du père et la cupidité cruelle des frères qui déchiraient la famille bien plus que le radicalisme des lois révolutionnaires. Catherine Douillat, originaire de Charente-Inférieure, accusait son frère de se montrer "plus attaché à la fortune que sensible à la tendresse que lui avaient toujours témoignée et sa mère et sa sœur ». Elle exigeait le maintien de la «juste» loi du 17 nivôse qui «mettait de l'égalité dans les familles en même temps qu'elle y maintenait l'union et la paix $»^{28}$.

La famille idéale : affection et égalité

24 Mais les femmes partisanes du divorce et du partage égal de l'héritage ne se bornaient pas à critiquer le système familial, elles s'efforçaient de créer le modèle d'une nouvelle éthique de la famille. Au moment même où, pour dénoncer la soumission des femmes au foyer, elles adoptaient l'idéologie des droits, les pétitionnaires s'apercevaient que la nouvelle éthique individualiste et égalitaire était en rupture avec un modèle plus ancien de participation à l'intérêt collectif. À la recherche d'un équilibre fragile, les pétitionnaires s'efforçaient de critiquer ces coutumes mais sans céder du terrain quant à la moralité. Tout en revendiquant l'égalité des droits dans la famille, elles construisaient une image alternative de familles unies par des liens d'affection. Pour démasquer les injustices courantes et valider leurs propositions de changement, elles avaient recours à un discours moral bien enraciné. Les principes politiques révolutionnaires ne leur apparaissaient pas simplement comme une bataille philosophique concernant les droits individuels des citoyens mais comme un affrontement moral quant à la définition de la justice au sein de la collectivité, c'est-àdire au sein de la famille et de la nation. Dans leur démarche pour remodeler la famille, les pétitionnaires, contrairement à ce qu'on pourrait attendre, ne se bornaient pas à créer un modèle domestique républicain et à mettre en valeur le rôle des femmes en tant que mères. Les accents de Rousseau sur la douceur maternelle se retrouvaient rarement dans leurs textes. Elles avaient plutôt tendance à forger une nouvelle vision de la famille fondée sur les liens d'affection et d'égalité également répartis "sans distinction de sexe ».

Certaines que la loi était apte à régénérer les émotions et la moralité, ces femmes proposaient une vision nouvelle et positive des liens familiaux. Les partisans du divorce 
reprenaient l'argument courant dans les brochures sur la question : l'existence même d'une loi encouragerait fidélité, tendresse et respect mutuel entre époux. La femme Lefebvre promettait aux législateurs que "par l'effet du divorce [...] les parents échappent à l'abîme de la débauche, et peuvent même augmenter leur bien-être par un mariage qui réunisse l'utile à l'agréable». C'est ainsi que «la liberté a le privilège exclusif d'épurer les mœurs». La femme Berlin répétait que la liberté de divorcer encouragerait toutes les épouses à rester fidèles et chaque mari à s'abstenir de traiter sa femme « en esclave, et il ne se croira pas le grand seigneur $»^{29}$.

En défendant une relation conjugale plus équitable et plus respectueuse, les pétitionnaires insistaient sur les avantages de la réforme du point de vue de la justice et des sentiments. Certaines proposaient de modifier la législation relative à la propriété des époux, qui plaçait veuves et divorcées dans une situation financière précaire. Lorsque la citoyenne Le Balleur envoie à la Convention une requête légale complexe, destinée à protéger la dot des femmes au «juste prix», elle souligne l'inégalité des structures maritales qui autorisait "un mari ou plutôt un maître " à contraindre sa femme à accepter de vendre sa dot. Se nommant elle-même "l'exposante», elle déclare : « Elle soulève avec chagrin le voile qui couvre le despotisme marital ; mais [...] je réclame cette équité naturelle, supérieure aux formes, aux textes, aux opinions, aux circonstances [...] je suis persuadée, citoyens représentants, qu'au nom du peuple français, vous donnerez à mes filles l'exemple d'une exacte justice en décrétant ce que je demande $»^{30}$. En invoquant « un juste prix» et une "équité supérieure aux formes, aux textes [...]», Le Balleur utilisait le langage éloquent de la morale économique, tout en rappelant les députés à leurs devoirs dans la nation nouvelle.

Les partisans de l'égalité dans l'héritage étaient nombreux à penser que non seulement les lois révolutionnaires rétabliraient "l'égalité dans les familles» mais qu'elles encourageraient les relations de bienveillance et mettraient fin « au règne dominateur où les plus forts voudraient opprimer les plus faibles». Le respect mutuel entre les membres d'une famille était une loi qui relevait et de la nature et de la société, «car quoi qu'on en dise, il est dans la nature qu'un même père partage toute sa tendresse, tous ses soins, le produit entier de ses travaux et de son économie entre tous ses enfants, sans aucune distinction de sexe $\aleph^{31}$. Dans une famille égalitaire, affection et moralité l'emporteraient sur l'ambition et l'autorité. Comme l'expliquait la citoyenne Bureau, originaire du Midi : «Moins les lois accordent au despotisme, plus il reste de force au sentiment. Annoncez aux pères que leur principal empire doit être resserré dans l'autorité de leurs vertus, dans la sagesse de leurs leçons et les témoignages de leur tendresse [...] ils seront plus écoutés, plus aimés et plus respectés [...] ainsi l'union, les soins réciproques, l'amour filial, s'enrichiront de ce qu'aura perdu la domination ${ }^{32}$. De justes lois cultiveraient « un juste équilibre » au sein du foyer, déclaraient les sœurs Delié : « Là une femme aimable et vertueuse reçoit de son mari reconnaissant et juste la récompense de ses travaux et de ses soins. Ici une mère intéressée est forcée de transmettre à ses enfants la portion que son mari lui avait assurée dans sa succession $\Perp^{33}$. Au lieu de proposer une simple solution individualiste, les pétitionnaires considéraient les lois comme un encouragement à responsabiliser équitablement les membres de la famille. Une nouvelle éthique d'égalité et de respect mutuel entre les sexes se substituerait, pour le plus grand bonheur de la famille, aux codes de hiérarchie et de sacrifice issus d'un autre âge. 
Les pétitions mettaient en évidence la dimension politique des réformes sociales. Rapprochant explicitement famille et nation, elles affirmaient que la réforme de la famille répondait aux vœux du peuple souverain en contribuant à épauler la République. Les lois équitables sur la succession satisfaisaient « l'intérêt public » et « le vœu de la majorité du peuple souverain » en rendant "vingt-cinq individus heureux pour chaque frère dénaturé ", répandant ainsi « la joie dans les familles " ${ }^{34}$. Certaines pétitionnaires allaient jusqu'à suggérer que les structures familiales s'accordaient avec diverses formes de gouvernement. La citoyenne Mallet, par exemple, déclarait que les anciennes coutumes familiales normandes convenaient à la monarchie, mais que la République devait instaurer le partage égal de la succession « sans distinction de sexe ", rétroactive depuis le 10 août 1792 . Certains de ces parallèles ne manquaient pas de pittoresque : «On dit encore tout haut que vous allez nous donner un roi si vous rendez à l'héritier ses anciennes prérogatives ", prévenait une pétitionnaire, ajoutant que, telle une deuxième Charlotte Corday, elle irait jusqu'à « enhardir les puînés et les sœurs malheureuses à se rendre justice elles-mêmes parce que la loi ne s'est intéressée à elles qu'un moment ». En effet, les intérêts de la nation, de la nature et de la moralité ne faisaient qu'un. La jeune Adélaïde Durand affirmait avec vigueur que le partage égal des biens permettrait aux sœurs célibataires «de se marier et de remplir le destin si conforme au vœu de la Nature et à l'intérêt de la Nation " $^{35}$.

Il est intéressant de constater que les pétitionnaires mettaient en parallèle les familles ouvertes aux sentiments et régies par un père compatissant avec un État gouverné par des législateurs généreux et accessibles ${ }^{36}$. De même que les pétitions esquissaient un monde idéal peuplé de pères pleins de bonne volonté, elles harcelaient les législateurs pour que ceux-ci se mettent à l'unisson de tous les citoyens quels qu'ils soient sur le plan de la morale et de la sensibilité et pour qu'ils maintiennent le partage égal «en gage de votre affection» vis-à-vis de tous les citoyens de la République. Proclamant leur « confiance intime » envers les députés, certaines allaient jusqu'à les prier, en tant que « pères de la Nation » ou en tant que pères de famille compatissants de soutenir les lois égalitaires. D'autres ajoutaient une clause originale en invitant les députés à intervenir en cas d'échec ou d'errements de leur propre père. En 1795, Jeanne Gallien les exhortait en ces termes : " Déniez remplacer leurs malheureux pères et défendez-les contre l'injustice, la tyrannie et le despotisme des frères de Normandie [...] tous vos enfants n'ont-ils pas les mêmes droits à votre tendresse? ». Joséphine Letellier invitait pareillement les députés à l'aider à « être épouse [...] être mère et citoyenne [...] que ne m'est-il possible de faire passer dans vos mains le pouvoir que mon père a sur moi ? ${ }^{37}$. Les pétitionnaires dans leur majorité ne demandaient pas aux députés d'intervenir aussi directement dans la famille mais, d'une manière certaine, ils demandaient tous à l'État de reconnaître la justesse et l'indépendance de leur critique des structures familiales. La Révolution ne se bornait pas à fournir un discours et un contexte politique aux revendications de réforme; elle se posait en rivale sur le plan de l'autorité, en alliée institutionnelle, légale et morale contre la famille traditionnelle. S'aligner avec la nation pour réclamer la régénération de la famille, pour écrire « au nomde toutes les républicaines de la ci-devant province de Normandie » comme celle qui se proclamait «Julie [...] républicaine » revenait à trouver un levier politique pour contrebalancer le poids des réseaux familiaux. Une telle démarche exerçait une pression morale sur les députés, les incitait à exécuter leur promesse et à prendre en compte les paroles des citoyens « ou citoyennes » ajoutait la veuve Descages ${ }^{38}$. 
30 Se plaçant entre la famille traditionnelle et le nouvel État, les pétitions en faveur de l'égalité présentaient leurs exigences et leur idéal. Non, le divorce et le partage égal de l'héritage ne déchireraient pas le tissu serré de la famille. Bien au contraire, ces mesures attendriraient le cœur des pères et des maris et encourageraient des rapports de réciprocité naturelle et juste, fondés sur l'affection mutuelle et l'égalité entre les sexes. Ces familles respecteraient les règles de justice collective et constitueraient la fibre morale de la République. Il y allait donc de la responsabilité et de l'intérêt des représentants de l'État de promulguer la réforme de la famille.

31 Cette analyse de pétitions féminines visant la réforme de la famille devrait contribuer à nous faire mieux comprendre la nature de la politisation des femmes et de leurs revendications pendant la Révolution. Pour la majorité d'entre elles, se lancer dans la politique révolutionnaire n'équivalait pas simplement à embrasser une série de principes entièrement nouveaux. Il s'agissait plutôt de réaliser un équilibre complexe entre d'anciennes et de nouvelles allégeances. Elles considéraient la politique avant tout comme un combat pour la justice et pour l'économie morale de la famille et de la nation. Pour définir ces objectifs, les pétitions en faveur du divorce et du partage égal de l'héritage s'emparaient du discours révolutionnaire de l'égalité des droits garantis par la nature et par la loi. Mais elles associaient cette idéologie excessivement individualiste à des notions morales profondément ancrées pour se livrer à une critique, radicale à l'occasion, des relations familiales existantes et de la différence des sexes. Il s'agissait aussi d'appeler à mettre la réciprocité des sentiments au centre de la moralité familiale et de lier la réforme de la succession et du mariage à la régénération de la famille et de l'État. De bonnes lois, déclaraient ces femmes, atteindraient cet objectif. Elles affirmaient que la nouvelle nation ne serait effective qu'à partir du moment où les droits des femmes ne seraient plus sacrifiés à la famille. Cet ensemble de philosophie des droits et de discours moral allait imprégner la pensée féministe en France au XIX siècle $^{39}$. À court terme, l'influence étonnante de cette idéologie au cours de la décennie révolutionnaire devait exacerber le conservatisme du Code civil. Il faut reconnaître que ce mouvement pétitionnaire animé et percutant se déroulait parallèlement à toute une activité de contestation juridique. Il arrivait souvent que les nouvelles règles du divorce sapent l'autorité du mari. De même, la nouvelle politique relative à l'héritage, ainsi que les procès se heurtaient aux stratégies familiales fixées depuis longtemps ainsi qu'à la domination des pairs et à la prépondérance des frères, en particulier des frères aînés. La résistance à ces nouvelles pratiques était générale et tenace car les lois, les institutions et l'idéologie révolutionnaire introduisaient brutalement dans la famille un nouveau modèle d'égalité entre individus et même entre les sexes. En même temps, l'expérience des procès s'associait à l'idéologie révolutionnaire, aux discours sur les droits et aux nouvelles pratiques politiques pour donner à certaines femmes le courage de démasquer l'inégalité de sexes dans la dynamique de pouvoir des familles et de faire connaître à leurs représentants, leurs revendications.

32 L'activisme des femmes dans le rôle de pétitionnaires ou de plaideurs révèle la complexité des effets de la Révolution sur les femmes. Plutôt que de se contenter de les exclure de la sphère publique, pour les reléguer dans le domaine privé, la Révolution mettait en question la nature de la famille, sans toutefois résoudre la question de la relation des femmes à la politique et à la famille. Dans les faits, la famille devient un lieu de conflit culturel et politique dans la mesure où le politique englobait tout 
l'espace, du public au privé. Pétrie de contradictions, la politique révolutionnaire laissait derrière elle un héritage conflictuel en matière d'inégalité entre les sexes. Sans doute, la Révolution stimula une vigoureuse argumentation en faveur du rôle domestique des femmes. Mais elle fit promulguer des lois leur accordant en tant qu'individus, de nouveaux droits civiques, de nouvelles formes d'accès à l'État, tout en créant un langage et des pratiques propres à critiquer les inégalités de genre. Même si les femmes n'avaient pas acquis la pleine participation politique pendant la Révolution, on constate que la démocratie républicaine ne se fondait ni sur un simple contrat sexuel ni sur une sphère privée qui restreindrait l'activité des femmes. Les effets de ces changements dans le domaine des lois, des institutions et de la culture politique variaient énormément selon la région, les structures familiales et sociales et la situation individuelle de chacune dans la famille. Il n'est pas possible d'évaluer globalement l'expérience qu'eurent les femmes de la Révolution. Mais il est clair que la Révolution créa des ouvertures et des possibilités situées à la charnière de la famille et de la nation, permettant à certaines femmes de se lancer dans l'activisme politique et juridique et d'imaginer une structure familiale marquée par des rapports plus égalitaires et plus affectueux. Ces actions démontrent bien la complexité du cheminement vers le rôle domestique des femmes, notion simultanément encouragée et sapée par la culture, les lois et les institutions révolutionnaires.

\section{NOTES}

1.AN DIII, pétition de la citoyenne Heringue aux représentants du peuple français, 10 floréal an III (20 avril 1795); AN D111 33, Pétition de la citoyenne LeFranc à la Convention nationale, 30 germinal an III (22 mars 1795).

2.Fondée sur la lecture de centaines de pétitions en faveur des droits civiques envoyées par des hommes et des femmes aux assemblées et aux comités révolutionnaires, Archives nationales (AN) séries AD II (Archives imprimées), AD XVIIIc (Suppléments aux Procès-verbaux des Assemblées nationales), C (Adresses à la Convention), DIII (Comité de législation); DXXXIX (Comité de la classification des lois).

3.Décret sur l'égalité des héritages en général du 17 nivôse an II et décret pour les enfants naturels du 12 brumaire an II.

4.Joan B. LANDES, Women and the Public Sphere in the Age of the French Revolution, Ithaca, N.Y., 1988 ; Madelyn GUTWIRTH, The Twilight of the Goddesses : Women and Representation in the French Revolutionary Era, New Brunswick, N.J., 1992 ; Dorinda OutRAM, The Body and the French Revolution, New Haven, 1989 et « Le langage mâle de la vertu : Women and the Discourse of the French Revolution, » dans The Social History of Language, ed. Peter BURKE and Roy PORTER, Cambridge, Eng., 1987. p. 120-135 ; Lynn HUNT, The Family Romance of the French Revolution, Berkeley, 1992.; Joan Wallach ScotT, Only Paradoxes to Offer : French Feminists and the Rights of Man, Cambridge, Mass., 1996, chap. 1-2 ; Geneviève FRAISSE, Muse de la raison. La démocratie exclusive et la différence des sexes, Aix-en-Provence, 1989. Carole PATEMAn, The Sexual Contract, Stanford, 1988 ; Michèle BordeAuX, "L'universalisme juridique et l'impasse de l'égalité », dans Les femmes et la Révolution 
française, 3 vols. (éd.) ; Marie-France BRIvE, Toulouse, 1989, 1, p. 427-440. Cf. Vida AzIMI, "L'exhérédation politique de la femme par la Révolution », Revue historique de droit français et étranger, 69, 1961, p. 177-216.

5.Des modèles de pétition attaquant les lois sur le partage égal de l'héritage étaient largement diffusés dans le Midi et en Normandie.

6.AN, D III 273, Pétition de la citoyenne LeBlanc aux citoyens représentants, 4 nivôse an II (24 décembre 1793) ; AN, DIII 33, Pétition de la citoyenne Marie Anne Angélique Fouque aux citoyens représentants, 24 vendémiaire an III (15 octobre 1794).

7.Alors que la question de l'héritage suscitait de nombreuses pétitions collectives, je n'en ai trouvé que très peu sur le divorce. Voir aussi Jean-Louis HALPERIN, L'impossible Code Civil, Paris, 1992, p. 215-220.

8.Les épouses et mères étaient bien plus nombreuses à écrire des pétitions au nom de leurs maris et de leurs fils que les maris et pères au nom de leurs épouses et filles.

9.AN, DIII 274, Pétition anonyme à la Convention nationale, signée par une "Calvadosienne », 12 messidor an III (30 juin 1795) ; AN, DIII 338, Pétition de la citoyenne Jeanne Barinçon au citoyen président de la Convention nationale, 26 prairial an III (14 juin 1795).

10.AN, DIII 32, Pétition des citoyennes du département du Calvados à la Convention nationale, r. 31 janvier 1793, signée par 15 femmes au nom d'« un grand nombre d'autres citoyennes qui ont déclaré ne savoir signer, se joignent à nous pour solliciter la même justice. » AN, DIII 338, Pétition des citoyennes du département de la Manche au citoyen président de la Convention nationale, envoyé de Coutances, 8 décembre 1792 ; AN, DIII 146, Pétition de plusieurs filles de Saint-Lô, à Monsieur le Président de l'assemblée législative, 5 juillet 1792 ; AN, DIII 338, Réclamation à la Convention nationale par des citoyennes des communes du district de Falaise (Calvados), s.d., vers floréal an III (mai 1795).

11.AN, C278, Lettre de la citoyenne Gavot à la Convention nationale, 1ère décade brumaire an II (oct. 1793) ; AN, DIII 273, Pétition de la citoyenne Geneviève Lebourdais femme Gras-Soubès au Comité de législation, 10 prairial an II (29 mai 1794).

12.AN, DIII 361, Pétition du citoyen Gauthier aux citoyens législateurs, s.d. (vers 1793-94) ; Pétition anonyme, s.d. (après 20 septembre 1792) ; Copie d'une lettre du citoyen Robin à sa femme, envoyée à l'assemblée, r. 21 décembre (1791 ou 1792) ; Pétition anonyme à l'Assemblée législative, reçue 4 octobre (sans année).

13.Sur les pamphlets de divorce rédigés par des hommes et des femmes, voir Suzanne DESAN, The Family on Trial in Revolutionary France, Berkeley, Cal., 2004, ch. I. Pour les reproductions de cahiers et de brochures de femmes publiés, voir 1789 Cahiers de doléances des femmes et autres textes, Paris, 1981 ; Les femmes dans la Révolution française, 3 vols., Paris, 1982 ; Réclamations des femmes 1789, Paris, 1989.

14.AN, DIII 273, Pétition de la citoyenne LeMachais au Comité de Législation, renvoyée le 5 brumaire an II (26 octobre 1793), Pétition de la citoyenne Félicité Desrogues femme Letellier au Comité de Législation, reçue le 23 vendémiaire an III (14 octobre 1794) ; AN, DIII 272, Pétition de la citoyenne R. Andaille au citoyen président de la Convention, 24 frimaire an II (14 décembre 1793); AN DIII 361, Pétition de la citoyenne Berlin à Monsieur le président de la Convention nationale, 27 septembre 1792. 15.Pétition de la citoyenne Th. Van Houten au Comité de Législation, s.d. vers 1793. 16.Suzanne DeSAN, Family on Trial, op. cit., p. 99-112 ; Francis Ronsin, Le contrat sentimental : Débats sur le mariage, l'amour, le divorce : de l'Ancien Régime à la Restauration, Paris, 1990, p. 271-274 ; Dominique DESSERTINE, Divorcer à Lyon sous la Révolution et 
l'Empire, Lyon, 1981, p. 188-194 ; Elaine KRUSE, « Divorce in Paris 1792-1804 : Window on a Society in Crisis » (Ph.D. thesis : University of Iowa, 1983), ch. 4 ; Roderick PHILLIPS, Family Breakdown in Late Eighteenth-Century France : Divorces in Rouen 1792-1803, Oxford, 1980, p. 56-58 ; Jean LHOTE, Le divorce à Metz et en Moselle sous la Révolution et l'Empire, Metz, 1981, p. 11-20.

17.AN, DIII 34, Pétition de la citoyenne Jacquette au Comité de Législation, 17 thermidor an III (4 août 1795).

18.AN, DIII 361, Pétition d'Anne Catherine Bagot à l'Assemblée nationale en faveur d'une femme délaissée depuis douze ans par son mari, reçue 31 janvier 1792.

19.AN, DIV 13, Pétition de Dutemple à Monsieur, 25 décembre 1790 ; AN, DIII 382, Projet de loi par François Belloc, homme de loi prés le tribunal du district de Tonneins, au Comité de Législation, 20 septembre 1793 ; Pétition anonyme à l'Assemblée nationale, s.d. ; AN, DIII 32, Pétition des grenadiers et volontaires du premier bataillon du Calvados aux législateurs, reçue le 19 mars, 1793.

20.«Remonstrances des mères et filles normandes de l'ordre du tiers », Rouen, 1789. Sur l'identité des auteurs, Claire LEFOLL, Les femmes et le mouvement révolutionnaires à Rouen, 1789-1795 (Mémoire de maîtrise, Université de Haute-Normandie, 1985), p. 45-46. 21.AN, DIII 35, Pétition de la citoyenne Marie-Marguerite Cordier femme Morin au Comité de Législation, 8 floréal an III (27 avril 1795) ; AN, DIII 272, Pétition d'Adélaïde Dorothée Durand à la Convention nationale, reçue le 2 ventôse an II (20 février 1794) ; AN, DIII 145, Pétition de la citoyenne Marie Anne Catherine Duquesnoy, fille majeure, au Comité de Législation, s.d. ; AN, DIII 382, Mémoire (par une femme anonyme) au Comité de Législation, s.d. : AN, DIII 338, Pétition de quatre veuves au Comité de Législation, 14 floréal, an III (3 mai 1795).

22.AN, DIII 338, pétition de la citoyenne Marguerite Briançon aux citoyens représentant, 14 floréal an III (3 mai 1795) ; Pétition de la citoyenne Piard Convers aux citoyens représentants, 12 germinal an III (1 avril 1795) ; AN, DIII 75, Pétition des citoyennes Bonelly (3 sœurs) au Comité de Législation, 16 floréal an III (5 mai 1795). 23.Sur la « sensibilité », Hans-Jurgen LÜSEBRINK, « L'innocence persécutée et ses avocats : Rhétorique et impact public du discours "sensible" dans la France du XVIII siècle ", Revue d'histoire moderne et contemporaine 40, 1993, p. 86-101; Sara MAZA, Private Lives, Public Affairs : The Causes célèbres of Pre-Revolutionary France, Berkeley, 1993 ; Lynn HunT, Family Romance, op. cit.

24.AN, DIII 274, Pétition de la citoyenne M. M. Anquetil aux citoyens représentants, 29 prairial an III (17 juin 1795) ; AN, DIII 32, Pétition des citoyennes soussignées du département du Calvados à la Convention nationale, reçue le 3 mars 1793, signée par 35 femmes et plusieurs $X$; c'est l'une de plusieurs pétitions collectives de groupes qui se désignent comme « citoyennes du Calvados ».

25.Suzanne DESAN, Family on Trial, op. cit., p. 150-165.

26.AN, DIII 273, Mémoire par Marie-Magdeleine Montfreulle, envoyé au Comité de Législation, 14 messidor an III (2 juillet 1795) ; AN, DIII 34, Pétition de la citoyenne Beaufils Veuve Lavallée au Comité de Législation, 9 messidor an II (27 juin 1794). 27.Les filles mariées dans la ci-devant province de Normandie au Corps législatif (Paris, s.d., vers an III).

28.AN, DIII 308, Pétition de la citoyenne Jeanne Côme aînée, aux représentants, pères d'un peuple libre, 14 floréal an III (3 mai 1795) ; Pétition de Catherine Douillat au Comité de Législation, reçue le 15 prairial an III (4 juin 1795). 
29.AN, DIII 361, Pétition de la citoyenne Degay, femme Lefèbvre aux législateurs, 10 septembre 1792 ; Pétition de la citoyenne Berlin à Monsieur le président de la Convention nationale, 27 septembre 1792.

30.Pétition de la citoyenne Marguerite Jeanne LeBalleur veuve Fiquet au Comité de Législation, 14 fructidor an III (31 août 1795).

31.AN, DIII 338, Pétition de Julie Poursent aux citoyens composant le Comité de Législation, n.d. ; Pétition de la citoyenne Jeanne Françoise Aumont au Comité de Législation, 7 messidor an III (25 juin 1795) ; Remonstrances des mères et filles normandes ; AN, DIII 339, Pétition de la citoyenne Ruette au Comité de Législation, 22 ventôse an III (12 mars 1795) ; AN, DIII 48, Question relative au décret du 8 avril 1791 sur les successions (Paris, s.d., vers An IV/1796).

32.Pétition de la citoyenne C. M. H. Bureau au Comité de Législation, reçue le 9 pluviôse an III (28 janvier 1795).

33.AN, DIII 271, Pétition des citoyennes Julie, Florence, et Cécile Délié au Comité de Législation, 28 messidor an II (16 juillet 1794). Cf. AN, DIII 143, Pétition de la citoyenne Létolé aux vertueux représentants, 29 ventôse an III (19 mars 1795) ; AN, DIII 147, Pétitions de la citoyenne Galot Thiphaine aux citoyens représentants, 3 \& 10 messidor an II (21 \& 28 juin 1794).

34.AN, DIII 338, Pétition de Marie Girard femme Leglize aux citoyens représentants, reçue le 29 floréal an III (18 mai 1795) ; AN, DIII 33, Pétition des citoyennes Marie Jeanne Rogne et Marie Rogne au Comité de Législation, 5 messidor an II (23 juin 1794) ; AN, DIII 146, Pétition de la citoyenne Marguerite Letellier femme LeMonnier aux représentants, 7 floréal an III (26 avril 1795).

35.AN, DIII 34, Pétition de la citoyenne Mallet au citoyen président de la Convention nationale, reçue le 11 pluviôse an II (30 janvier 1794) ; AN, DIII 338, Pétition de la fille Villier aux citoyens législateurs, 16 floréal an III (5 mai 1795) ; AN, DIII 272, Pétition d'Adélaïde Dorothée Durand, à la Convention nationale, reçue le 2 ventôse an II (20 février 1794).

36.Lynn Hunt, Politics, Culture, and Class in the French Revolution, Berkeley, 1984, p. 42-43, 72-74.

37.AN, DIII 32, Pétition des citoyennes Alexandre, LeTonneur, et Antoine aux citoyens députés, reçue le 2 mai 1793 ; AN, DIII 143, Pétition de la citoyenne Jeanne Gallien veuve Barenton aux citoyens représentants, 9 floréal an III (28 avril. 1795) ; AN, DIII 147, Pétition de Josephine Letellier aux citoyens législateurs, 26 pluviôse an II (14 février 1794).

38.AN, DIII 382, Pétition de la citoyenne Julie [...] républicaine au citoyen président de la Convention nationale, reçue le 1 novembre 1793 ; AN, DIII 338, Pétition de la citoyenne Rausan veuve Descages aux citoyens [représentants], 27 floréal an III (16 mai 1795).

39.Karen OfFEN, « Defining Feminism : A Comparative Historical Approach », Signs : Journal of Women in Culture and Society 14, 1988, p. 119-157 ; Joan SCOTT, Only Paradoxes to Offer: French Feminists and the Rights of Man, Cambridge, Mass., 1996, Ch. 3. 


\section{RÉSUMÉS}

Au cours de la Révolution, des centaines de femmes adressèrent des pétitions aux assemblées nationales pour réclamer des modifications du droit de la famille, tel que le droit au divorce et au partage égal de l'héritage. Les pétitionnaires utilisaient souvent la rhétorique révolutionnaire afin de critiquer le «despotisme» masculin régnant dans la famille et de plaider pour des rapports familiaux plus égalitaires et plus affectueux. Notre article met en question l'idée selon laquelle la Révolution se bornait à encourager le rôle domestique des femmes à l'intérieur de la «sphère privée ». Son action était plus ambiguë. Tout en soutenant fermement la rhétorique de la domesticité, la Révolution octroya aux femmes des droits civiques ainsi qu'un accès plus facile aux gouvernants et créa le langage et la pratique politique nécessaires à la critique des inégalités de genre.

The Petitions of Women in Favor of Revolutionary Reform of the Family. During the French Revolution, hundreds of women addressed petitions to the national assemblies to demand modifications in family rights like the right of divorce and an equal share in inheritance. The petitioners often used revolutionary rhetoric to criticize male «despotism» reigning in the family, and to plead for more egalitarian and more affectionate family relationships. This article challenges the notion that the Revolution was limited to encouraging the domestic role of women within the «private sphere». Its action was more ambiguous. While firmly supporting the rhetoric of domesticity, the Revolution granted women civic rights as well as easier access to those in political power, and created the language and political practice necessary for the criticism of gender inequalities.

INDEX

Mots-clés : divorce, égalité, héritage, autorité, despotisme

\section{AUTEUR}

\section{SUZANNE DESAN}

Professeur d'histoire, History Department, 455 N. Park St., University of Wisconsin, Madison, WI 53706 USA. 